\title{
Introduction: Decompressive craniectomy for trauma and cerebrovascular disease
}

\author{
Geoffrey T. Manley, M.D., Ph.D. \\ Department of Neurological Surgery, University of California, San Francisco, California
}

\begin{abstract}
Elevated intracranial pressure is one of the most common causes of death and disability following severe traumatic brain injury and ischemic stroke. Unfortunately, there have been no new medical treatments for cerebral edema and elevated intracranial pressure in more than 80 years. Decompressive craniectomy may be an appropriate surgical option in the face of elevated intracranial pressure that is refractory to medical treatment. When performed correctly, this procedure can reduce intracranial pressure and prevent cerebral herniation and death. The last decade has seen a renewed interest in the use of decompressive craniectomy, but many questions remain regarding patient selection, timing of surgery, surgical technique, timing of cranioplasty, and complications.
\end{abstract}

This issue of Neurosurgical Focus serves to summarize some of the latest findings and presents new data in decompressive craniectomy. In the first article, Danish et al. report their use of meta-analysis to examine outcome and quality of life in patients after decompressive craniectomy and their finding of improved outcome compared to studies published 30 years ago. This is followed by 3 articles that describe the successful use of decompressive craniectomy in patients with cerebral ischemia secondary to cerebrovascular disease. These studies suggest a wider indication for this procedure beyond ischemic stroke and begin to tackle important questions regarding the use of this surgical technique in the elderly. From a technical perspective, Kenning et al., compare a "hinge" craniotomy to the standard decompressive craniectomy. Their paper highlights the need to study more technical aspects of this procedure. Stiver, importantly, reviews the complications of decompressive craniectomy in traumatic brain injury, and Aarabi et al. describe the interesting phenomena of subdural hygromas following this procedure. The timing of cranioplasty following successful recovery from decompressive craniectomy is controversial. Gooch et al. explore this issue in a single institution with interesting results. Finally, Cabraja and colleagues report their experience and long-term results using titanium implants for correcting large cranioplasty defects.

25.

Together these articles provide timely and much needed information regarding decompressive craniectomy for trauma and cerebrovascular disease. I hope that you will find this issue of Neurosurgical Focus interesting and that it will serve to stimulate future studies. 